\title{
Une coopération antinomique
}

Le cas italien

An antinomic relationship - The Italian experienceUna cooperación antinómica.

El caso italiano

\section{Paola Dusi}

Traducteur : Carine Drouet

\section{(2) OpenEdition}

\section{Journals}

Édition électronique

URL : https://journals.openedition.org/ries/1873

DOI : 10.4000/ries. 1873

ISSN : 2261-4265

Éditeur

France Education international

Édition imprimée

Date de publication : 1 décembre 2002

Pagination : 39-50

ISBN : 978-2854-2-0555-8

ISSN : 1254-4590

\section{Référence électronique}

Paola Dusi, « Une coopération antinomique », Revue internationale d'éducation de Sèvres [En ligne], 31 décembre 2002, mis en ligne le 24 novembre 2011, consulté le 21 septembre 2021. URL : http:// journals.openedition.org/ries/1873; DOI : https://doi.org/10.4000/ries.1873 


\title{
Une coopération antinomique *
}

\section{Le cas italien}

\section{Paola Dusi}

\begin{abstract}
"L'excès de normativité dans le domaine de l'éducation produit un regroupement abstrait de ceux qui ne sont pas impliqués dans les processus éducatifs et les intègre dans un système de compétition. L'abstraction consiste dans le fait que les normes de l'école sont appliquées d'une part sans références aux besoins et intérêts des personnes auxquelles elles s'appliquent, et sont d'autre part isolées de leur expérience et de leur système de relations» (Habermas 1989 : 371).
\end{abstract}

Avec le changement de contexte socio-culturel, politique, axiologique et économique, et même ethnique du vieux continent, dans la toute fin du $\mathrm{XX}^{\mathrm{e}}$ siècle, on est passé de «l'euphorie» à la «désillusion» vis-à-vis de l'institution scolaire ${ }^{1}$. L'écart entre les objectifs proclamés dans les documents officiels de l'école et la réalité de la vie en classe et de la société semble se creuser. La baisse de la démographie, en partie compensée par l'arrivée de noyaux familiaux «autres», et la diffusion des nouvelles technologies de l'information ont inévitablement modifié le milieu de vie de l'homme occidental. À cela s'ajoute le fait que de tels changements «constituent des défis pour les écoles et leurs curricula à un moment où les écoles elles mêmes souffrent d'une réduction de financements et où les conditions qui ont garanti leur stabilité sont fondamentalement en train de changer. ${ }^{2}$ L'altération des caractéristiques traditionnelles propres au marché du travail, aux typologies professionnelles, à l'expérience relative au travail a sensiblement modifié les attentes de la communauté envers le système scolaire.

\footnotetext{
* Cet article a été traduit par Carine Drouet.

1. T. Husén, The School in Question, London, 1979.

2. R. Meighan, W. Reid, «How Will the "New Technologies" Change The Curriculum?» in Journal of Curriculum Studies, 4, 1982, London, p. 353 et suivantes.
} 


\section{LA CRISE DU SYSTÈME SCOLAIRE}

Dans la société de la nouvelle économie, des sites Internet, de l'inversion des flux migratoires du sud vers le nord, de la globalisation économique sinon culturelle, les requêtes que les apprenants, les familles et la société même adressent à l'école et à ses opérateurs ont changé. Le changement, et de la structure occupationnelle, et du concept même d'occupation, la recherche axiologique croissante, la possibilité de satisfaire l'instruction en dehors de l'école, une incidence et importance mineures de l'action de formation de l'école (comme lieu et agent de préparation spécifique ${ }^{3}$ ne sont que certains des sujets qui rendent nécessaire une réflexion sur le rôle de l'école dans une société marquée par de rapides changements. Déjà, dans les années 80 , un document OCDE-CERI signalait que, fréquemment, la perception sociale du système scolaire était centrée sur la faible efficacité de ce dernier. En particulier, la formation était définie selon deux types de représentation antagoniques : celle "de l'élitisme» et celle "de l'égalitarisme» ${ }^{4}$. Selon la première, l'école semble avoir pris le chemin de la médiocrité; pour ce qui est de la seconde, le système de formation semble incapable de contribuer au "rattrapage» des handicaps socioculturels. Dans un tel contexte, devant la nécessité de redéfinir les rôles et les devoirs de l'établissement scolaire, l'école doit avant tout, d'après Meighan et Reid, "cesser de faire des choses que d'autres peuvent mieux faire et s'occuper des buts de l'éducation ${ }^{5}$.

Ce qui est devenu évident pour tous, c'est, d'une part, la perte d'efficacité de l'école et, de l'autre, le besoin urgent de partager avec la famille, premier milieu éducatif, l'action de formation institutionnelle. Dans la société de la communication et de l'incertitude, il n'est plus possible de reconnaître à l'école un rôle d'exclusivité / centralité dans le parcours de formation des nouvelles générations. Par conséquent «la prétention de continuer à penser à l'instruction scolaire comme quelque chose d'autosuffisant et d'achevé (...) n'a plus de raison d'être. Il est indispensable de s'habituer à considérer le curriculum de l'école comme une expérience "conditionnelle", c'est-à-dire dépendante des conditions culturelles extérieures à l'école et influencée par elles, et l'expérience même de l'école comme "provisoire" dans son ensemble, autrement dit relative, pas déci-

3. «L'instruction a été construite autour du noyau essentiel de l'apprentissage à lire, écrire et compter, qui introduisait la valeur instrumentale de la culture en la transformant en une valeur avec des finalités sociales et politiques; à ces valeurs, aujourd'hui, on pourrait ajouter celles des langues et des compétences informatiques. La formation concentre l'attention vers le développement des capacités du sujet avec une finalité heuristico-constructive pour sa connaissance même et pour la qualité de son expérience; la formation générale accentue ainsi le déplacement de l'attention vers les compétences générales du sujet : expérimenter, connaître, évaluer, juger, décider, etc. On pourrait rappeler ici aussi la position de V. E. Frankl, lequel considère que la dynamique interne du processus de croissance est constituée et guidée par la catégorie générale de l'auto-transcendance. » L. Guasti, «Progetto educativo per un sistema formativo integrato », Pedagogia e Vita,1983, 3, p. 135.

4. OCDE-CERI, Quality in Education, Paris, 1983.

5. R. Meighan, W. Reid, «How Will the "New Technologies” Change The Curriculum ? » p. 356-358. 
sive dans l'absolu, impliquant un certain degré d'inefficacité par rapport à la totalité des questions qu'on lui pose, intégrable de différentes façons » ${ }^{6}$.

Cette situation de profonde inquiétude concernant le système de formation formel ${ }^{7}$ s'est développée - et pas seulement dans notre pays - à partir des années 60, en concomitance avec le phénomène de la scolarisation de masse. Cette récente et incontestable conquête des démocraties occidentales a été, en effet, marquée par une crise de confiance lorsqu'on a pris conscience que l'école dispense des certifications fondamentalement disqualifiées tant sur le plan qualitatif que par rapport au marché du travail. En outre, «la prédominance d'une culture formalisée, à la fois sujet et objet de certification des études réalisées, et aussi nécessaire à l'appropriation par chacun du patrimoine des produits de l'esprit humain (culture patrimoine ${ }^{8}$ ), a apporté avec elle une progressive homogénéisation culturelle, calquée sur des modèles standard, renforcés par la culture diffuse des mass media, qui ont comme effacé la culture entendue au sens socio-ethno-anthropologique (culture d'émancipation ${ }^{9}$ ) avec tout ce que chaque culture a de spécifique : modèles de vie, valeurs, traditions, langages ${ }^{10}$. En disant cela, on ne veut certainement pas nier le rôle de premier plan que l'école occupe dans la vie de chacun, celui d'une société fondée sur le respect de l'individu, de la liberté et de la démocratie. Et pourtant, face à des attitudes d'insatisfaction et de critique de plus en plus répandues dans l'opinion, il apparaît non seulement inutile mais tout à fait néfaste de nier les limites et le manque d'efficacité qui affectent notre système de formation.

\section{LE PARTENARIAT ÉCOLE/FAMILLE : UN CADRE VIDE}

La réponse au mal-être avec lequel l'école «lit» l'imaginaire collectif et la communauté sociale se situe dans le cadre des réformes institutionnelles qui ont concerné tantôt les programmes, tantôt les modèles d'organisation, tantôt la structure institutionnelle du système de formation formel. On peut replacer dans cette perspective les mesures législatives de décentralisation connues sous le nom de «décrets de délégation». Avec l'approbation de la loi de délégation $\mathrm{n}^{\circ} 477$ du 30 juillet 1973 suivie du décret de délégation nº 416 du 31 mai 1974,

6. C. Scurati, I. Fiorin, Dai programmi alla scuola. Principi pedagogici e metodologici dell'azionedidattica, La Scuola, Brescia, 1997, p. 21-22. Voir aussi, E.J. King, «Education for a Communications Society », in E.J. King (ed.), Reorganizing Education, London, 1977, p.7-37; E.J. King, Education for Uncertainty, London, 1979.

7. À entendre comme système institutionnel consacré à l'élaboration et à la transmission de la culture même de chaque société ainsi qu'à la formation de ses acteurs en nombre croissant.

8. NDT : en français dans le texte.

9. NDT : en français dans le texte.

10. C. Scaglioso, «Una nuova certificazione di qualità », in G. Dalle Fratte (a cura di), Autonomia della scuola e sviluppo formativo, Trento, Uno edizioni, 1994, p. 144. 
l'école italienne sembla laisser derrière elle le modèle de centralisme pour devenir «l'école de la communauté» ${ }^{11}$.

Le texte législatif ${ }^{12}$ reconnaissait formellement aux parents un espace dans le cadre de la structure scolaire; le changement de perspective dans la gestion de l'école a été d'une telle portée qu'il a été accueilli comme une mesure à même de rendre concret le concept de «démocratie effective». Dans les décrets de délégation, "le thème de la participation de la famille à la vie de l'école sert de ligne de partage entre une façon d'être marquée par l'étroitesse empirique, le caractère unidirectionnel des intervenants, l'exaltation de la composante informative de l'école, et un projet de développement marqué par la réciprocité de propositions et d'actions entre école et famille. Tout cela donc, dans le but d'ouvrir l'école au milieu environnant, principalement à la famille, et d'améliorer la définition du projet éducatif de la communauté locale. ${ }^{13}$

L'ouverture de nouveaux espaces d'intervention qui impliquait et légitimait l'interaction entre les deux agents de formation, école et famille, n'a pas tardé à se révéler un cadre vide, en trahissant les attentes de ceux qui reconnaissent dans la participation de la famille et de toute la communauté un aspect valorisant du processus éducatif. Les raisons de la perplexité et de la désillusion qui ont bien vite accompagné la mise en place de la participation parentale au système de formation peuvent être synthétiquement déterminées par le faible pouvoir attribué aux "organes collégiaux» et par l'impréparation des acteurs impliqués : enseignants et parents. Les uns ont démontré qu'ils ne possédaient

11. L. Corradini, La difficile vie commune. De l'école d'État à l'école de la communauté, La Scuola, Brescia, 1975. Déjà, en 1970 , le ministère de l’Instruction publique avait promulgué la circulaire ministérielle $n^{\circ} 375$ qui fondait les comités école - famille : première tentative «formelle» de reconnaître aux parents le rôle qui leur est propre en tant que dépositaires du droit premier de l'éducation.

12. Le cadre de la participation des parents à ces structures internes à l'école qui ont comme fonction la réglementation, la coordination, la supervision et le contrôle itinérant, a été repris et défini avec l'acte législatif $n^{\circ} 297$ de 1994. L'article 5, «conseil d'intersection, d'interclasse et de classe » annonce : «font aussi partie du Conseil d'intersection, d'interclasse ou de classe : a) dans les écoles maternelles et les écoles primaires, pour chacune des sections ou des classes intéressées, un représentant élu par les parents des élèves inscrits; b) dans les écoles secondaires (collèges), quatre représentants élus par les parents des élèves inscrits dans la classe; c) durant les cinq dernières années de l'enseignement secondaire italien (lycée), deux représentants élus par les parents des élèves inscrits dans la classe, ainsi que deux représentants des élèves, élus par ceux-ci. À l'article 8, alinéa 1,6,7, « conseil d'administration ou d'établissement et commission exécutive », la prescription législative établit que le conseil d'administration ou d'établissement, dans les écoles avec une population scolaire allant jusqu'à 500 élèves, est constitué de 14 membres, dont 6 représentants du personnel enseignant, un du personnel administratif, technique et auxiliaire, 6 parents d'élèves, le directeur d'école ou le proviseur; dans les écoles avec une population scolaire supérieure à 500 élèves, il est constitué de 19 membres, dont 8 représentants du personnel enseignant, 2 du personnel administratif, technique et auxiliaire, 8 parents d'élèves, le directeur d'école ou le proviseur. Dans les instituts d'enseignement supérieur (universités), les représentants des parents d'élèves sont réduits, par rapport à la population scolaire, à 3 et à 4 ; dans ce cas là ils sont appelés à faire partie du conseil autant que les représentants élus par les étudiants. Le conseil d'administration ou d'établissement est présidé par un des membres, élu à la majorité absolue (de ses membres), parmi les représentants des parents d'élèves. La promotion de la participation de la famille à la vie scolaire est, en outre, garantie par : des entretiens individuels avec des enseignants ou des consultants; des assemblées de classe, de "plesso» (terme bureaucratique qui désigne une structure fonctionnelle constituée d'un ensemble d'organes qui développent des activités semblables), d'administration ou d'établissement; des commissions relatives aux aspects spécifiques de la vie scolaire et des comités de parents.

13. L. Pati, La politica familiare nella prospettiva dell'educazion, La Scuola, Brescia, 1995, p. 336-337. 
pas la maturité professionnelle nécessaire à l'accueil d'une telle mesure; les autres ont fait preuve de préjugés, d'une faible capacité de proposition, au point d'introduire dans l'école des divergences de nature idéologique et politique qui ont rendu presque impossible le dialogue avec les enseignants. Les organes collégiaux, donc, "ont formellement constitué une certaine avancée dans la perspective de la construction de la communauté scolaire, mais ne sont pas parvenus, concrètement, à abattre les murs qui séparent encore l'école de la société civile et de la culture produite, diffusée et consommée à l'extérieur ${ }^{14}$. Corradini, à ce sujet, parle d'un «énorme gâchis d'espoirs et de potentiel éducatif ».

La reconnaissance d'un rôle aux parents dans la gestion de l'école, avec leur participation aux organes auxquels incombe la direction de chaque école (le conseil d'intersection pour l'école maternelle; le conseil d'interclasse et le conseil d'administration pour l'école primaire; le conseil de classe et le conseil d'établissement pour l'école secondaire), s'est révélée être une expérience malheureuse, d'autant que dans les années 80 , la désaffection des parents envers les organes collégiaux s'est accrue de façon exponentielle. Il s'agit, en effet, d'une participation de nature en général formelle, vide et non finalisée à tel point qu'au cours de cette période s'est accrue dans les faits la distance entre le dispositif législatif prévu par le décret de délégation et la capacité, la volonté et l'engagement des personnes appelées à le mettre en œuvre ${ }^{15}$. En résumé, donc, s'il faut reconnaître aux décrets de délégation le mérite d'avoir ouvert les portes de l'institution scolaire à la famille et à la société, «l'école de la communauté» ne semble pas prête à arriver dans notre pays.

\section{Une ÉCOLE AUTONOME}

L'impulsion innovatrice qui, dans les années 90, a investi toute la société italienne dans sa tentative de stopper le processus de progressive détérioration politique, culturelle et sociale, a déterminé dans l'autonomie un «nouveau principe régulateur d'efficacité et de vigueur du système (...), qui est un élément de croissance et de développement d'une démocratie effective (...), lequel valorise et met au premier plan la personne et ses communautés et promeut bien plus le développement des identités culturelles et sociales que ce que peut faire la présence, pourtant nécessaire, du système formel de règles et de procédures démocratiques, qui ne génère pas une réelle pratique fondée sur une véritable participation ${ }^{16}$ En 1997, dans le cadre d'un processus qui

14. C. Scaglioso, Una Nuova Certicazione della qualità, p. 144.

15. L. Corradini, «I nessi tra famiglia e scuola e l'associazionismo familiare, in campo scolas tico », in P. Donati (ed), Terzo rapporto sulla famiglia in Italia, Milano S. Paolo, 1993, p. 197-201.

16. G. Dalle Fratte, «Il Manifesto dell'autonomia », in G. Dalle Fratte (ed), Autonomia della Scuola e sviluppo formativo, p. 16-17. 
implique tout un aménagement social, on introduit dans l'institution scolaire le concept d'autonomie (mesure législative $\mathrm{n}^{\circ} 59$, art.21) : «la réforme du système d'instruction et de formation a trouvé dans le concept d'autonomie le contenu autour duquel focaliser sa propre stratégie». ${ }^{17}$ Ceci afin de mettre en pratique l'instance de la coopération école / famille, en proposant des formes de contrôle réel de la collectivité sur l'école, sans toutefois léser la compétence ni le professionnalisme des enseignants. Selon ces principes, le système de formation formel doit se structurer comme organisation parallèle et indépendante par rapport au système politique et au système économique, et par là même éviter les entraves de la bureaucratie et satisfaire les demandes de participation, les exigences de démocratie et de pluralisme présentes dans l'environnement social. Le concept d'autonomie, en effet, n'est pas entendu seulement comme modalité d'organisation, mais plutôt comme expression de la complexité qui marque la société contemporaine et, surtout, comme "catégorie constitutive» d'une «communauté scolaire» au sens plein du terme. La pluralité et l'autonomie des institutions et des associations formelles et informelles de citoyens sont un corollaire naturel du concept de démocratie; en l'occurrence, il s'agit d'impliquer les acteurs jusqu'alors restés en marge du système scolaire : élèves, parents, représentants des administrations locales ${ }^{18}$.

Le «règlement en matière d'autonomie des établissements scolaires" reconnaît formellement le rôle exercé par les parents à l'intérieur de la structure scolaire. Le texte stipule : " le plan d'offre de formation est élaboré par le conseil des enseignants sur la base des tendances générales pour les activités de l'école et des choix de gestion et d'administration définis par le conseil d'administration ou d'établissement, compte tenu des parents, des propositions et des opinions formulées par les organismes et les associations, et par les étudiants pour les universités (...). Le plan d'offre de formation est rendu public et transmis aux élèves et aux familles lors de l'inscription ». ${ }^{19}$ Il est clair qu'il n'est pas encore possible de parler d'une co-responsabilité des acteurs impliqués dans

17. L. Guasti, «L'autonomia del sistema pedagogico », in Pedagogia e Vita, 1999, 2, p. 139. L'article 21 de la mesure législative $n^{\circ} 59 / 97$ (qui esquisse la réforme générale de l'administration publique) « développe certains aspects de la mesure de 1974, à même d'assigner à l'école plus d'espaces culturels et opérationnels, en lui donnant une plus grande responsabilité conceptuelle et créatrice de projets». L. Guasti «Considerazioni sul rapporto tra organizzazione e didattica nella legge 'Bassanini' », in Pedagogia e Vita, 1998, 4, p. 132.

18. "La riche pluralité et l'autonomie d'initiative culturelle des formations sociales - comme sujets responsables, capables d'activités, porteurs de leur spécificité, protagonistes de leur histoire - demandent au système et aux structures politiques reconnaissance, promotion et garanties de développement». G. Dalle Fratte, "Il manifesto dell'autonomia», p. 15.

19. D.P.R. 275/99, Regolamento in materia di autonomia delle istituzioni scolastiche. Le plan d'offre de formation est le document que chaque institution scolaire, de tout ordre et de tout degré, fait obligatoirement rédiger pour le conseil des enseignants, avec la supervision du dirigeant scolaire au début des cours. Un tel document est présenté aux parents dans le cadre des rencontres collégiales qui sont programmées (ex : conseil d'interclasse). Le POF correspond et s'identifie avec le curriculum de formation de l'école d'origine; par conséquent, on doit y spécifier les activités curriculaires et extra-curriculaires que l'établissement entend réaliser pour cette année scolaire en particulier. 20. P. Pasotti, «I genitori negli organi collegiali dell'autonomia », in La Famiglia, 1997, 186. 
la gestion du système scolaire. Toutefois, «dans l'actuelle phase de redéfinition des rôles et des responsabilités, le rapport école / famille revient à assumer une position centrale avec toutes les implications culturelles, politiques, pédagogiques, et sociales correspondantes $» .{ }^{20}$ Dans l'attente d'une réforme des organes collégiaux prévue par la loi 59/97, la participation de la famille à la vie de l'école est précisée et spécifiée par le concept de coopération introduit par l'article 1 de la loi 30/2000. «La coopération indique le rôle actif des parents, leur concours en termes opérationnels dans le domaine de l'instruction / éducation scolaire ». ${ }^{21}$ Avec l'énonciation formelle du concept de coopération, l'exigence d'une implication concrète des parents dans la gestion du projet éducatif devient explicite: une présence qui saurait aller plus loin que la participation formelle (délégation et exercice du vote) et effective (intervention aux réunions et aux assemblées) jusqu'alors prévue par la réglementation ${ }^{22}$ en vigueur, dont les modalités qui n'ont pas donné lieu à une réelle coresponsabilité éducative des acteurs du système de formation, à la fois à l'intérieur et à l'extérieur de l'établissement scolaire. Les traditionnels moments de dialogue parents - enseignants n'ont pas suffi pour dépasser les méfiances réciproques et les stéréotypes réducteurs en permettant d'élaborer un projet éducatif partagé. L'école autonome doit donc reconnaître à la famille le rôle qui lui revient en tant qu' «artisan fondamental d'éducation " ${ }^{23}$ dans l'individualisation des besoins, dans la détermination des stratégies et dans le contrôle du système d'éducation / instruction.

\section{UNE COOPÉRATION UTOPIQUE?}

Les nombreuses études qui ont été menées sur le thème des relations école / famille ont depuis longtemps démontré comment la participation parentale à la gestion du parcours de formation a des répercussions positives sur la réussite scolaire des enfants / élèves. Les parents, depuis les années 80, ont porté une attention croissante à la vie scolaire de leur progéniture. Un changement significatif, en particulier, a été relevé dans l'attitude des parents à l'égard de l'institution scolaire. On est passé, lentement mais de façon irréversible, d'une attitude de déférence, conditionnée par un sens d'inadéquation et d'impuissance, à une attitude fondée sur la prise de conscience de leurs droits et leurs devoirs à l'égard du système de formation. Les enseignants, pour leur part, déplorent à la fois l'absence des parents du parcours scolaire des enfants et un

21. L. Pati, «Famiglia e scuola per l'educazione : dal partecipare al cooperare», in La Famiglia, 2002, 211, p. 19.

22. Les normes qui régissent la participation de la famille au système scolaire sont les suivantes : Ordonnance ministérielle $n^{\circ} 215$ du 15/07/1991: «Élection des organes collégiaux au niveau de l'administration-établissement; Acte législatif 297 du 16/04/1994: "Texte unique des mesures législatives en matière d'instruction, relatives aux écoles de tout ordre et de tout degré »; Ordonnance ministérielle n² 277 du 17/06/1998: «Élection des organes collégiaux»; Acte législatif 233 du 30/06/1999: "Réforme des organes collégiaux territoriaux de l'école».

23. L. Pati, «Famiglia e scuola per l'educazione : dal partecipare al cooperare», p. 18. 
intérêt centré uniquement sur les résultats obtenus (notes ou appréciations, réussite ou échec), enfin la prédominance d'une attitude de délégation de leurs responsabilités au système d'instruction / éducation auquel il est demandé de suppléer à l'action de la famille. Tout en reconnaissant que la collaboration entre école et famille représente une condition sine qua non pour la définition d'un parcours de formation de qualité, parents et enseignants peinent à laisser derrière eux préjugés et défenses corporatives, à apprendre les uns des autres dans le respect des rôles réciproques, dans la perspective du «bien-être» des enfants et donc de toute la société. Les parents, dépositaires du droit premier à l'éducation de leur progéniture ${ }^{24}$, et les enseignants, titulaires de compétences et de professionnalisme, sont appelés à apprendre l'art de la rencontre, de la confrontation et du dialogue ${ }^{25}$ dans un rapport d'égalité respectueux de la particularité de chaque instance éducative. Il faut éviter confusion et/ou homologation de fonctions pédagogiques, dans l'optique de la complémentarité des rôles. Il est demandé aux parents et aux enseignants, en dernier ressort, de "coopérer» c'est-à-dire de partager : a) la responsabilité et le pouvoir de la gestion du système éducatif, mais pas nécessairement à part égale; b) projets et objectifs du parcours de formation, même si chacun contribue de façon différente à leur réalisation en fonction de la spécificité pédagogique de leur rôle; c) l'engagement dans une action commune afin que «les choses marchent».

La coopération apparaît donc comme l'instrument privilégié, peut-être utopique $^{26}$, pour parvenir à un projet éducatif précis et partagé par les élèves, parents, enseignants, par la communauté d'appartenance, dans le but d'une éducation de qualité en mesure de former des personnalités harmonieuses et responsables envers elles-mêmes et envers les autres. Ceci implique soit la définition de principes d'orientation généraux (pour garantir le respect des valeurs communes de citoyenneté, sauvegarder le droit à l'instruction, l'intérêt commun et l'équité), soit la garantie d'une pluralité de parcours possibles (pour sauvegarder la responsabilité, la liberté de choix, la diversité). Déterminer des valeurs communes respectant de l'originalité de chacun et du groupe dans lequel ce dernier se reconnaît, ne représente certes pas une tâche facile, même pour des lieux, comme l'école et la famille, destinés à l'exercice du respect, de la liberté, de l'amour et de la connaissance.

24. Droit établi par des documents officiels comme la Déclaration universelle des droits de l'homme, «Les parents ont le droit préférentiel à choisir le type d'éducation que devront recevoir leurs enfants» (art.3), et comme la Constitution italienne, «ll est du devoir et du droit des parents de subvenir aux besoins, instruire et éduquer leurs enfants; même s'ils sont nés hors mariage » (art. 30, alinéa 1).

25. La rencontre met en cause le professionnalisme de l'enseignant et l'identité éducative des parents. La confrontation repose sur l'examen des contenus éducatifs exprimés par les deux instances éducatives et se concrétise seulement si elle est maintenue par l'intentionnalité des partis. Le dialogue ne prend de valeur que lorsque les attitudes critiques sont abandonnées au profit d'une définition des objectifs communs que doit viser l'action éducative. L. Pati, La funzione educativa del padre, Vita e Pensiero, Milano, 1981, p. 211-221.

26. Le concept d'utopie n'est pas pris dans le sens de «lieu qui n'existe pas» mais plutôt dans celui de «lieu pas encore atteint ». 
Dans un contexte socioculturel connoté par une fragmentation sociale en augmentation, marqué par la présence d'individus et de familles qui appartiennent à différentes cultures, parvenir à un accord sur les valeurs auxquelles on ne peut renoncer et qui fondent le parcours de formation, sera certainement plus ardu en raison du fait que des valeurs, essentielles et pas toujours négociables, sont en jeu. La rencontre avec autrui, dont la diversité prend des formes plus ou moins floues, oblige à repenser le sens de notre façon de vivre, la signification de notre savoir-être. L'altérité, avec sa différence, implique inévitablement le conflit mais est aussi l'occasion de percevoir sa propre originalité et de devenir authentiquement soi-même grâce à un dialogue "antinomique » avec la figure de l'autre. Un autre qui est lu, pensé, «jugé » souvent sur la base de son appartenance ethnique, culturelle, politique, de genre, de classe et de rôle. L'hostilité, le refus, qui pour une bonne part ont rendu plus difficiles les relations famille / école, sont souvent une conséquence directe d'une lecture préconçue et stéréotypée du rôle de l'autre, d'une opposition des parties : famille contre école, école contre famille ${ }^{27}$. Pour cette raison, il apparaît indispensable, entre autres choses, de repenser la formation des enseignants et de projeter des parcours éducatifs également pour les parents, de manière à ce qu'ils trouvent des moments communs afin de permettre à chacun de connaître le point de vue et les raisons de l'autre, et d'apprendre l'exercice correct des instruments que la démocratie scolaire met à disposition.

La coopération dialectique semble par conséquent être l'unique voie qui laisse place pour l'homme au futur et au respect. Famille et école sont appelées à apprendre à vivre ensemble pas seulement en vue de la formulation d'un projet éducatif apte à satisfaire les exigences des élèves, des parents, des enseignants, de la communauté et du marché du travail, mais surtout parce qu'elles constituent les lieux destinés à enseigner aux nouvelles générations l'art d' "apprendre à vivre ensemble en développant une compréhension des autres et une appréciation de l'interdépendance (en réalisant des projets communs et en apprenant à gérer les conflits), dans un esprit de respect des valeurs du pluralisme, de la compréhension réciproque et de la paix ${ }^{28}$ La coopération famille / école interroge, en dernier ressort, non seulement le cadre normatif qui définit également l'espace du dialogue et du conflit "constructif» entre les acteurs de la co-responsabilité éducative souhaitée, mais aussi notre manière d'être des personnes, des hommes et des femmes, des pères et des mères, des parents et des enseignants à l'intérieur d'une collectivité démocratique.

27. «D’une meilleure conscience de l'importance des stéréotypes et des préjudices, des émotions et de leurs dérivés affectifs, pourrait naître une sagesse honnête et sereine au service de l'amélioration de la vie. (...) Les intérêts, les égoïsmes, les rumeurs, la confusion, les orgueils opposés pourraient aussi conduire à la perte... » E. Tiberi, Il primato delle emozioni, Giuffrè, Milano, 1988, p. 260.

28. J. Delors, Nell'eucazione un tesoro, Armando Editore, Roma, 2000, p. 89. 


\section{RÉFÉRENCES BIBLIOGRAPHIQUES}

Ouvrage collectif (1990): L'educazione del cittadino. Ricerche sulla formazione socio-civico-politica, La Scuola, Brescia, parte Ia.

Ouvrage collectif (1969): Scuola e Famiglia, Vita e Pensiero, Milano.

Ouvrage collectif (1975): La nuova scuola della partecipazione, Milano, Vita e Pensiero.

Ouvrage collectif (1998): Sussidiarietà e nuovi orizzonti educativi : una sfida per il rapporto famiglia-scuola, Brescia, La Scuola.

CAPPABIANCA G. (1997): «L'organizzazione della scuola tra Stato e autonomia», in AA. VV., Teorie pedagogiche e dimensioni professionali, Brescia, La Scuola, pp. 254-259.

CATTANEO M. (2000) : «Famiglia e Scuola», in Scuola Materna, 6, pp. IX-X.

PATI L. (1997): "Famiglia e Scuola per ridare l'anima alla società », in Pedagogia e Vita, 4, pp. 127-135.

CECCATTONI R. (2002) : «La collaborazione tra scuola e famiglia : insieme per il bambino ", La Famiglia, 212, pp. 76-84.

CORRADINI L. (1978): Scuola e famiglia, A. Armando, Roma.

CORRADINI L. (1975): La difficile convivenza. Dalla scuola di Stato alla scuola della comunità, La Scuola, Brescia.

CORRADINI L. (1986): La scuola e i giovani verso il Duemila. Problemi di educazione etico-politica, Teramo, Giunti-Lisciani Editori.

CORRADINI L. (1993): «I nessi tra famiglia e scuola e l'associazionismo familiare in campo scolastico", in P. DONATI (a cura di), Terzo rapporto sulla famiglia in Italia, Milano, S. Paolo, pp. 197-201.

CORRADINI L. (1986): «Famiglia e partecipazione sociale», in AA. VV., Famiglia e Società, Edizioni del Rezzara, Vicenza, pp. 191-204.

CHIOSSO G. (1992): «Sviluppo e declino della scuola italiana», in ACONE G., BERTAGNA G., CHIOSSO G., Paideia e qualità della scuola, La Scuola, Brescia, pp. 61-74.

CSONKA L. (1973): Scuola e famiglia, Roma, Bulzoni.

DAMIANO E. (a cura di) (1992): Professionalità e partecipazione comunicativa. Una ricerca educativa nella scuola materna, Brescia, La Scuola.

DE GIORGI P., MALIZIA G., MONNI G., STENCO B. (2002): «La cooperazione educativa della famiglia nella scuola cattolica/1", in La Famiglia, 212, pp. 29-38.

DE GIORGI P., MALIZIA G., MONNI G., STENCO B. (2002): «La cooperazione educativa della famiglia nella scuola cattolica/2», in La Famiglia, 213, pp. 18-29. 
DURNING P. (ed) (1988): Éducation familiale. Un panorama des recherches internationales, Paris, Éditions Matrice.

DURNING P. (1995) : Éducation familiale. Acteurs, processus et enjeux, Paris, PUF.

DURNING P., POURTOIS J.-P. (1994): Éducation et famille, De BoeckWesmel, Bruxelles.

EPSTEIN J.L. (1987): "Toward a theory of family-school connections : Teacher practices and parent involvement» in K. HURRELMANN, F. KAUFMAN, F. LOEL (Eds), Social Intevention : Potential and Constraints, Walter de Gruyter, New York.

EPSTEIN J.L. (1996) : "Family-school links : How do they affect educational outcomes?», in A. BOOTH, J. DUNN (Eds), Family-School Links : How do they affect educational outcomes?, Lawrence Erlbaum Associates, Hillsdale, NJ.

FRABBONI F., GENOVESI G. (1990): La scuola e $i$ suoi problemi. Per una teoria della scuola, La Nuova Italia, Firenze, pp. 67-81.

FRANCESCHETTI F., FRANCESCHETTI F. (1969): «Rapporto scuolafamiglia : contestazione o collaborazione da parte dei genitori?», in AA. VV., Scuola e famiglia, Milano, Vita e Pensiero.

GALLI N. (1975) : "La partecipazione dei genitori alla gestione sociale della scuola", in AA. VV., La nuova scuola della partecipazione, Milano, Vita e Pensiero.

GAYET D. (1999): L'école contre les parents, INRP, Parigi.

GRASSILLI B. (1994) : «Rapporto scuola/famiglia fra formalismo partecipativo e istanze di rinnovamento ", in AA. VV., Essere famiglia oggi, Quaderni dell'Agesc Lombardia, Brescia, pp. 43-54.

GUASTI L. (1998) : "Considerazioni sul rapporto tra organizzazione e didattica nella legge "Bassanini" », in Pedagogia e Vita, 4, pp. 123-133.

GUASTI L. (1999) : «L'autonomia del sistema pedagogico», in Pedagogia e Vita, 2, p. 141.

GUASTI L. (1998): "Progetto educativo per un sistema integrato", in Pedagogia e Vita, 3, pp. 126-136.

GUASTI L. (1998): Curricolo e riforma della scuola, Brescia, La Scuola, pp. 43-60.

HENRIOT-VAN ZANTEN A. (1988): «Les familles face à l'école. Rapports institutionnels et relations sociales ", in P. DURNING (ed), Éducation familiale. Un panorama des recherches internationales, Paris, Éditions Matrice. LUCCIO R. (1974): Famiglia e scuola nel processo educativo, Bologna, Il Mulino.

MACARIO L., SARTI S.: Scuola e famiglia, Zürich, Pas-Verlag, 1971. 
MONNIER R., POURTOIS J.-P. (1987): Relations école-famille. Formation des maîtres, Labor, Bruxelles, pp. 47-76.

MONTANDON C. (1994): "Les relations parents-enseignants dans l'école primaire : de quelques causes d'incompréhensions mutuelles", in DURNING P., POURTOIS J.-P., Éducation et famille, De Boeck-Wesmel, Bruxelles, pp. 185-200.

OCDE- CERI (1983): Quality in Education, Paris.

OCDE (1997): Les parents partenaires de l'école, Paris.

PERUCCI C.: «La presenza della famiglia nella scuola come dimensione essenziale dell'educazione», in AA. VV., Scuola e famiglia.

PASOTTI P. (1997) : "I genitori negli organi collegiali dell'autonomia», in La Famiglia, 186, pp. 51-52.

PASOTTI P.(2000): «I genitori e il POF : verso nuovi rapporti tra scuola e famiglia », in La Famiglia, 203, pp. 60-61.

PATI L. (1997): "Famiglia e scuola per ridare l'anima alla società», in Pedagogia e Vita, 4, pp. 131-132.

PATI L. (2001): «Famiglia e scuola per l'educazione : dal partecipare al cooperare », in La Famiglia, 205, pp. 15-26.

POURTOIS J.-P. (1997) : "Trasformazioni nelle relazioni tra scuola e famiglia», in Rivista Quadrimestrale dell'Istituto Superiore Internazionale Salesiano di Ricerca Educativa (ISRE), 2.

POURTOIS J.-P. et Alii (1984): Éduquer les parents ou comment stimuler la compétence en éducation, Bruxelles, Éditions Labor.

REGUZZONI M.: «La partecipazione delle comunità naturali alla gestione della scuola», in AA. VV., Scuola e famiglia.

RIVA A. (1972) : Famiglia e scuola, Roma, Coines.

ROSSI B. (1997) : «Scuola e famiglia nella prospettiva della reciprocità », in Studium Educationis, 3, pp. 396-398.

STIEVENHART M. (1989): "L'émergence d'une notion : le partenariat socio-éducatif», in Les Sciences de l'éducation pour l'ère nouvelle, 5, pp. 3550 .

ZAY D. (1994) : «Partenariat», in Dictionnaire encyclopédique de l'éducation et de la formation, Nathan, Paris, pp. 721-724. 